\title{
Chip-Spread CDMA: uma variação da transmissão DS-CDMA extremamente eficiente como técnica de acesso múltiplo
}

\author{
Emilio Rodríguez-Hernández e Raimundo Sampaio-Neto
}

\begin{abstract}
Resumo-Este artigo enfoca uma técnica de acesso múltiplo que combina a transmissão em blocos com portadora única e a técnica de multiplexação CDMA. Esta combinação referida como Chip Spread Code Division Multiple Access (CS-CDMA) resulta em uma técnica diversa da tradicional DS-CDMA e apresenta sobre esta última vantagens que incluem uma significativa superioridade de desempenho. A adoção de faixa de guarda Zero Padding sugerida e analisada neste artigo em substituição ao clássico Cyclic Prefix, mostrou-se capaz de melhorar sensivelmente o desempenho da transmissão CS-CDMA. Além disso, como medida da eficácia desta técnica, é feita uma comparação de desempenho com a técnica Orthogonal Frequency Division Multiple Access (OFDMA) indicada para a adoção nos sistemas 5G. Os resultados expressos em termos da taxa de erro de bit indicaram uma expressiva superioridade do sistema CS-CDMA.
\end{abstract}

Palavras-Chave-Acesso Múltiplo, Chip Spread CDMA, OFDMA, desempenho.

Abstract-This work focuses on a multiple access technique that combines single-carrier block transmission and the CDMA technique. This combination referred to as Chip Spread Code Division Multiple Access (CS-CDMA) results in a technique different from the traditional DS-CDMA and has shown benefits over this last technique that include a significant superiority in performance. The adoption of a Zero Padding type of guard interval, proposed and analyzed here, in lieu of the classical Cyclic Prefix, resulted in an expressive improvement in the performance of the CS-CDMA transmission. Furthermore, as a measure of the effectiveness of this technique, this article presents a performance comparison with OFDMA multiple access technique, indicated for adoption in the 5G standards. The bit error rate results evidenced a very expressive superiority of the CS-CDMA method.

Keywords-Multiple Access, Chip-Spread CDMA, OFDMA, Performance.

\section{INTRODUÇÃO}

Os sistemas de comunicações que são afetados por canais multipercursos, tais como os sistemas celulares podem sofrer importantes limitações do desempenho. Os níveis da interferência entre símbolos (IES) constituem um efeito preocupante para os sistemas convencionais de transmissão sequencial. Uma alternativa para minorar essa limitação é encontrada nos sistemas de transmissão em blocos, que sofrem da interferência entre blocos (IEB), mas que pode ser evitada com a inserção de

Emilio Rodríguez-Hernández e Raimundo Sampaio-Neto, Centro de Estudos em Telecomunicações (CETUC), Pontifícia Universidade Católica do Rio de Janeiro (PUC-Rio), Rio de Janeiro, RJ 22451-900, Brasil, e-mail: emilio@cetuc.puc-rio.br, raimundo@cetuc.puc-rio.br. Este trabalho foi parcialmente financiado por CNPq (132571/2019-6). um intervalo de guarda. Após a adição do intervalo de guarda o bloco de símbolos resultante pode ser transmitido utilizando um esquema de transmissão em múltiplas portadoras ou em portadora única.

Cada nova geração de comunicações móveis busca uma melhora com relação à anterior quanto à capacidade de transmissão de dados, à eficiência, à portabilidade, entre outros. À cada transição os requisitos se tornam mais exigentes fazendo com que a comunidade cientifica procure novas alternativas para encontrar soluções inovadoras. Com o objetivo de ampliar a capacidade da comunicação dos sistemas celulares para suportar cada vez mais usuários, novas formas de aproveitar os recursos do canal são pesquisadas. A transmissão simultânea de dados sobre um mesmo canal é uma forma viável de aproveitamento dos recursos de espectro, viabilizada pela utilização de diferentes esquemas de múltiplo acesso. Todas as gerações de comunicações móveis têm utilizado alguma técnica de multiplexação baseada em divisão no tempo, divisão na frequência, divisão em códigos ou combinações delas [1]. De forma geral os esquemas de múltiplo acesso podem-se classificar como: ortogonais e não ortogonais. Os esquemas de múltiplo acesso ortogonais exploram recursos ortogonais como janelas de tempo disjuntas reservadas para os diferentes usuários, diferentes portadoras ou códigos ortogonais, permitindo a separação dos usuários na recepção. As técnicas ortogonais vêm sendo muito utilizadas inclusive na rede atual $4 \mathrm{G}$.

$\mathrm{Na}$ literatura se podem encontrar diversos trabalhos que combinam diferentes técnicas de acesso múltiplo, como $\mathrm{Di}$ rect Sequence-Code Division Multiple Access (DS-CDMA) combinado com Orthogonal Frequency Division Multiplexing (OFDM), para obter novos esquemas de acesso múltiplo [2]. Técnicas com portadoras múltiplas como Multicarrier CDMA (MC-CDMA) [2] e Multicarrier DS-CDMA [3], [4] também foram propostas visando atingir melhores desempenhos. Uma desvantagem importante que afeta os sistemas com portadoras múltiplas é o elevado Peak-to-Average Power Ratio (PAPR) [5], o que motivou o estudo de abordagens que empregam sistemas com portadora única para a transmissão dos blocos de dados [5], [6], [7].

Este artigo enfoca uma técnica que combina a transmissão em blocos com portadora única e a técnica de multiplexação CDMA. Esta combinação, referida como Chip-Spread Code Division Multiple Access (CS-CDMA) resulta em uma técnica diversa da tradicional DS-CDMA e apresenta sobre esta última diversas vantagens incluindo uma alta superioridade de desempenho [8], [9]. Como medida de eficácia desta técnica, é feita 
neste artigo uma comparação de desempenho com a técnica de múltiplo acesso Orthogonal Frequency Division Multiple Access (OFDMA) [10] indicada para a adoção nos sistemas 5G. A comparação entre as duas abordagens considera sistemas com múltiplos usuários e transmissão através de canais com multipercursos.

Notação: Os caracteres maiúsculos em negrito representam matrizes e os caracteres minúsculos em negrito denotam vetores. Os operadores $(.)^{T},(.)^{H},(.)^{-1}$ representam a transposta, o hermitiano e a inversão de uma matriz, respetivamente. O operador $\mathbb{E}[$.$] denota o valor esperado.$

\section{SiSTEMAS DE TRANSMISSÃO EM BLOCOS}

Este artigo considera comunicação em configuração de uplink para um sistema multiusuário com $K$ usuários ativos. $\mathrm{O}$ canal de comunicações que conecta os usuários com a Estação Radio Base (ERB) é modelado por um filtro FIR (Finite Impulse Response) de comprimento $P$. Antes de cada transmissão é adicionado um intervalo de guarda de tamanho suficiente (não menor que $P-1$ ) para permitir eliminar a IEB na recepção. A seguir é apresentada a modelagem dos sinais de um sistema CS-CDMA e, para completude, a modelagem dos sinais do sistema OFDMA e o esquema de detecção considerado neste artigo.

\section{A. Modelagem da técnica CS-CDMA}

Nos sistemas CS-CDMA propostos em [8], diferentemente dos sistemas DS-CDMA, os chips que formam a sequência de código são espalhados pelo bloco de símbolos de informação, o que corresponde a um intercâmbio entre os símbolos de informação e os chips do código de espalhamento presentes na estrutura dos sistemas DS-CDMA tradicionais. A principal vantagem do CS-CDMA é que permite manter a ortogonalidade entre os sinais transmitidos pelos diferentes usuários, mesmo após a passagem por canais multipercursos e seletivos em frequência o que não ocorre nos sistemas DS-CDMA. Considerando uma transmissão síncrona para o caso do uplink com $K$ usuários ativos, o $k$-ésimo usuário transmite uma sequência de símbolos $b_{k}(i)$ que são pontos da constelação complexa de sinais do esquema de modulação empregado. Neste caso, como é considerada a transmissão em blocos, um bloco de $B_{0}$ símbolos de dados, $\mathbf{b}_{k}=\left[\begin{array}{llll}b_{k}(0) & b_{k}(1) & \ldots & b_{k}\left(B_{0}-1\right)\end{array}\right]^{T}$ é formado. No sistema CS-CDMA, este bloco de símbolos é multiplicado pelo $i$-ésimo chip pertencente à sequência de espalhamento de comprimento $T_{0}$ do $k$-ésimo usuário, $c_{k, i}$. A estrutura do sistema é mostrada na Figura 1 onde se observa que a duração da sequência transmitida pelo $k$-ésimo usuário é de $B_{0} T_{s}$ onde $T_{s}$ equivale à duração de um símbolo de dados [8].

O bloco transmitido no esquema CS-CDMA pelo $k$-ésimo usuário na $i$-ésima transmissão é dado por $\mathbf{b}_{k} c_{k, i} \operatorname{com}(i=$ $\left.0,1,2, \ldots, T_{0}-1\right)$. Após a inserção do intervalo de guarda com $L_{0}$ amostras no bloco $\mathbf{b}_{k} c_{k, i}$, os elementos do bloco resultante de comprimento $M_{0}=L_{0}+B_{0}$ são sequencialmente transmitidos através de um canal multipercurso com resposta impulsional $\mathbf{h}_{k}$ de comprimento $P$. A transmissão através do canal pode ser representada por uma matriz Teoplitz de

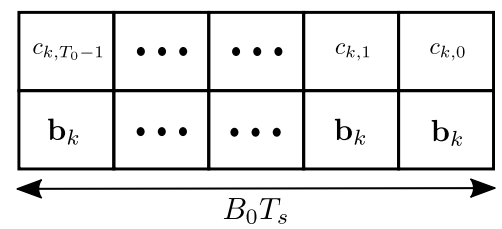

Fig. 1. Sequência de $T_{0}$ blocos transmitidos pelo $k$-ésimo usuário em CSCDMA.

convolução $\overline{\mathbf{H}}_{\mathbf{k}}$ de dimensão $M_{0} \times M_{0}$ cuja primeira coluna é dada pela resposta impulsional $\mathbf{h}_{k}$ concatenada com $M_{0}-P$ zeros. Na recepção pela ERB o processamento de detecção depende do tipo de intervalo de guarda empregado: prefixo cíclico ou zero-padding (ZP). Estes dois casos são abordados a seguir.

1) Prefixo Cíclico: Para o caso onde é utilizado o CP, após da remoção extensão cíclica para eliminação da IEB a matriz de convolução $\overline{\mathbf{H}}_{\mathbf{k}}$ se torna uma matriz circulante $\operatorname{Circ}\left(\overline{\mathbf{h}}_{k}\right)$ de dimensão $B_{0} \times B_{0}$, com a primeira coluna dado pelo vetor $\overline{\mathbf{h}}_{k}$, correspondente ao vetor $\mathbf{h}_{k}$ concatenado com $B_{0}-P$ zeros. Assim, para o caso da configuração uplink o sinal recebido pela ERB pode ser expresso como

$\overline{\mathbf{r}}_{C P}(i)=\sum_{k=1}^{K} \operatorname{Circ}\left(\overline{\mathbf{h}}_{k}\right) \mathbf{b}_{k} c_{k, i}+\overline{\mathbf{n}}_{C P}(i) \quad i=0,1, \ldots, T_{0}-1, s$

onde $\overline{\mathbf{n}}_{C P}(i)$ representa um vetor de ruído aditivo Gaussiano branco com média nula e matriz de covariância $\mathbb{E}\left[\overline{\mathbf{n}}_{C P}(i) \overline{\mathbf{n}}_{C P}^{H}(i)\right]=\sigma^{2} \mathbf{I}_{B_{0}}$. Como o código ortogonal correspondente ao $k$-ésimo usuário, $\left\{c_{k, i}\right\}_{i=0}^{T_{0}-1}$, é formado por $T_{0}$ chips, então são necessárias a recepção e coleta de $T_{0}$ blocos consecutivos, como ilustrado na Figura 1, obtendo-se uma matriz de observação, que levando-se (1) em consideração, pode ser expressa na forma

$\mathbf{R}_{C P}=\left[\overline{\mathbf{r}}_{C P}(0) \ldots \overline{\mathbf{r}}_{C P}\left(T_{0}-1\right)\right]=\sum_{k=1}^{K} \operatorname{Circ}\left(\overline{\mathbf{h}}_{k}\right) \mathbf{b}_{k} \mathbf{c}_{k}^{T}+\mathbf{N}_{C P}$,

onde $\mathbf{N}_{C P}=\left[\begin{array}{lll}\overline{\mathbf{n}}_{C P}(0) & \ldots & \overline{\mathbf{n}}_{C P}\left(T_{0}-1\right)\end{array}\right]$.

2) Zero-Padding: No caso de utilização do $Z P$, sugerida e analisada neste artigo, diferentemente do caso $\mathrm{CP}$, o intervalo de guarda não é suprimido na recepção. Cada um dos $T_{0}$ blocos de dados transmitidos é concatenado com um vetor de zeros de comprimento $L_{0}$ ao seu final, gerando o bloco de transmissão de tamanho $M_{0}$. A matriz Toeplitz de convolução $\overline{\mathbf{H}}_{\mathbf{k}}$ que caracteriza o canal do $k$-ésimo usuário tem dimensão $M_{0} \times M_{0}$, porém, a adição dos zeros no final do vetor de dados $\mathbf{b}_{k}$ permite que se considere apenas as primeiras $B_{0}$ colunas da matriz $\overline{\mathbf{H}}_{\mathbf{k}}$, formando a matriz $\overline{\mathbf{H}}_{\mathbf{k}}^{(\mathbf{0})}$ de dimensão $M_{0} \times B_{0}$ como nova representação do canal. Então a recepção no caso da configuração uplink o sinal recebido pela ERB pode ser expresso como

$$
\overline{\mathbf{r}}_{Z P}(i)=\sum_{k=1}^{K} \overline{\mathbf{H}}_{\mathbf{k}}^{(\mathbf{0})} \mathbf{b}_{k} c_{k, i}+\overline{\mathbf{n}}_{Z P}(i) \quad i=0,1, \ldots, T_{0}-1,
$$

onde $\overline{\mathbf{n}}_{Z P}(i)$ representa um vetor de ruído aditivo Gaussiano branco com média nula e matriz de covariância 
$\mathbb{E}\left[\overline{\mathbf{n}}_{Z P}(i) \overline{\mathbf{n}}_{Z P}^{H}(i)\right]=\sigma^{2} \mathbf{I}_{M_{0}}$. Concatenando os chips de forma semelhante ao caso do $\mathrm{CP}$, se pode gerar o vetor $\mathbf{c}_{k}$, obtendose uma matriz de observação formada por

$$
\mathbf{R}_{\mathbf{Z P}}=\left[\begin{array}{llll}
\overline{\mathbf{r}}_{Z P}(0) & \ldots & \overline{\mathbf{r}}_{Z P}\left(T_{0}-1\right)
\end{array}\right]=\sum_{k=1}^{K} \overline{\mathbf{H}}_{\mathbf{k}}^{(\mathbf{0})} \mathbf{b}_{k} \mathbf{c}_{k}^{T}+\mathbf{N}_{Z P},
$$

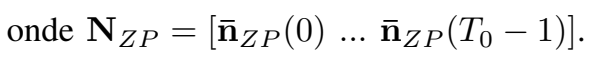

\section{B. Modelagem da técnica OFDMA}

A técnica de transmissão OFDM é uma técnica de transmissão multiportadora que surge para aliviar os problemas do sistema serial, que utiliza modulação com portadora simples. A principal vantagem de OFDM é a redução da sensibilidade do sistema ao espalhamento de retardo do canal, e portanto, à IES. Ao dividir o sinal de entrada em sub-bandas, cada subbanda irá experimentar um desvanecimento aproximadamente plano em frequência.

OFDMA consiste na atribuição de uma ou várias subbandas de frequência a um usuário, com a limitante que o espaçamento entre as sub-bandas seja o mesmo que o utilizado por OFDM de $1 / T_{s}$ para assegurar a ortogonalidade [10].

Para a modelagem de OFDMA se considera uma transmissão síncrona em configuração uplink com $K$ usuários ativos. O sistema depois de paralelizar o vetor de dados $\mathbf{d}=\left[\begin{array}{llll}d_{1} & d_{2} & \ldots & d_{N}\end{array}\right]^{T}$ e nele aplicar a Transformada Inversa de Fourier (IDFT), por meio da multiplicação pela matriz $\mathbf{W}_{N}^{H}$, adiciona o intervalo de guarda ao vetor resultante $\tilde{\mathbf{d}}$, tipicamente preenchido pelo prefixo cíclico $(\mathrm{CP})^{1}$, que incorpora as $L$ últimas amostras do vetor $\tilde{\mathbf{d}}$ ao início do bloco a ser transmitido. O bloco OFDMA transmitido tem portanto um comprimento de $M=N+L$. O sinal transmitido passa por um canal multipercurso modelado por um filtro discreto com resposta impulsional dada pelo vetor $\mathbf{h}_{P}=\left[\begin{array}{llll}h_{0} & h_{1} & \ldots & h_{P-1}\end{array}\right]^{T}$. Além do canal, o sinal transmitido é também afetado por ruído aditivo Gaussiano branco. A transmissão através do canal pode ser representada por uma matriz Toeplitz de convolução $\mathbf{H}$ de dimensão $M \times M$. Na ERB após remoção do $\mathrm{CP}$ a matriz $\mathbf{H}$ se torna uma matriz circulante, $\operatorname{Circ}(\mathbf{h})$ de dimensão $N \times N$ com a primeira coluna dada pelo vetor $\mathbf{h}$, correspondente ao vetor $\mathbf{h}_{p}$ concatenado com $N-P$ zeros.

A matriz normalizada de Transformada Discreta de Fourier (DFT) é representada por $\mathbf{W}_{N}$ tal que $\mathbf{W}_{N}^{H} \mathbf{W}_{N}=$ $\mathbf{W}_{N} \mathbf{W}_{N}^{H}=\mathbf{I}_{N}$. Suas componentes são dadas por:

$$
\left[\mathbf{W}_{N}\right]_{m, v}=\frac{1}{\sqrt{N}} e^{-j \frac{2 \pi}{N} m v} \quad 0 \leq m, v \leq N-1 .
$$

Após a demodulação com a matriz DFT, o sinal recebido no domínio da frequência no $i$-ésimo instante é dado por

$$
\mathbf{r}(i)=\mathbf{W}_{N} \operatorname{Circ}(\mathbf{h}) \mathbf{W}_{N}^{H} \mathbf{d}(i)+\mathbf{W}_{N} \mathbf{n}(i) .
$$

A pré- e pós-multiplicação pelas matrizes de DFT e IDFT, respectivamente, tornam a matriz circulante $\operatorname{Circ}(\mathbf{h})$ uma matriz diagonal, pelo fato de que qualquer matriz circulante

\footnotetext{
1 a transmissão CP-OFDMA com prefixo cíclico é a técnica tradicional e indicada para uso em sistemas $5 \mathrm{G}$
}

é diagonalizável pela operação da Transformada Discreta de Fourier.

$$
\mathbf{r}(i)=\operatorname{Diag}(\mathbf{q}) \mathbf{d}(i)+\mathbf{n}^{\prime}(i) .
$$

A diagonal da matriz resultante $\operatorname{Diag}(\mathbf{q})$ é formada pelas componentes da resposta de frequência do equivalente passabaixa discreto do canal

$$
\mathbf{q}=\sqrt{N} \mathbf{W}_{N} \mathbf{h}
$$

As estatísticas de um vetor Gaussiano branco não se alteram com a aplicação de uma transformação ortogonal como a feita por $\mathbf{W}_{N}$, portanto o vetor $\mathbf{n}^{\prime}(i)=\mathbf{W}_{N} \mathbf{n}(i)$ continuar sendo Gaussiano branco com média nula e matriz de covariância $\mathbf{K}_{n}^{\prime}=\sigma^{2} \mathbf{I}_{N}$. Assim os vetores $\mathbf{n}(i)$ e $\mathbf{n}^{\prime}(i)$ são estatisticamente equivalentes.

O desenvolvimento a seguir apresenta o modelo matricial para a configuração uplink:

1) Uplink ou enlace reverso: No enlace reverso os usuários realizam a transmissão e a ERB recebe a informação de parte de todos eles. Para exemplificar a modelagem adotada, é apresentado aqui um caso simples onde o sistema tem dois usuários ativos simultaneamente $(K=2)$. Primeiramente, os usuários começam a transmissão, onde cada um deles transmite pelo seu canal correspondente. Após a remoção da extensão cíclica as matrizes dos canais se tornam matrizes circulantes e o vetor recebido pela ERB pode ser expresso na forma:

$$
\begin{aligned}
\mathbf{y}= & \operatorname{Circ}\left(\mathbf{h}_{1}\right) \mathbf{W}_{N}^{H}\left[\begin{array}{llll}
\mathbf{d}_{1}^{T} & 0_{1} & \ldots & 0_{N-J}
\end{array}\right]^{T} \\
& +\operatorname{Circ}\left(\mathbf{h}_{2}\right) \mathbf{W}_{N}^{H}\left[\begin{array}{llll}
0_{1} & \ldots & 0_{N-J} & \mathbf{d}_{2}^{T}
\end{array}\right]^{T}+\mathbf{n}
\end{aligned}
$$

onde

$$
\mathbf{d}_{i}=\left[\begin{array}{llll}
d_{i 1} & d_{i 2} & \ldots & d_{i J}
\end{array}\right]^{T} i=1,2 \quad J \leq \frac{N}{2},
$$

$\mathbf{h}_{1}$ e $\mathbf{h}_{2}$ são as respostas ao impulso do equivalente passabaixa discreto dos canais correspondentes a cada usuário. Considerando a pré/pós multiplicação pelas matrizes DFT e IDFT, a matriz circulante se torna uma matriz diagonal, resultando em

$$
\begin{aligned}
\mathbf{r}= & \operatorname{Diag}\left(\mathbf{q}_{1}\right)\left[\begin{array}{llll}
\mathbf{d}_{1}^{T} & 0_{1} & \ldots & 0_{N-J}
\end{array}\right]^{T} \\
& +\operatorname{Diag}\left(\mathbf{q}_{2}\right)\left[\begin{array}{llll}
0_{1} & \ldots & 0_{N-J} & \mathbf{d}_{2}^{T}
\end{array}\right]^{T}+\mathbf{n}^{\prime}
\end{aligned}
$$

onde $\mathbf{q}_{1}$ e $\mathbf{q}_{2}$ são os coeficientes das transformadas das respostas ao impulso discreta dos canais como em (8). A expressão (11) pode ser reescrita como

$$
\mathbf{r}=\operatorname{Diag}(\mathbf{q})\left[\begin{array}{ll}
\mathbf{d}_{1} & \mathbf{d}_{2}
\end{array}\right]^{T}+\mathbf{n}^{\prime},
$$

onde

$$
\operatorname{Diag}(\mathbf{q})=\left[\begin{array}{cc}
\operatorname{Diag}\left(\mathbf{q}_{1}^{\prime}\right) & 0 \\
0 & \operatorname{Diag}\left(\mathbf{q}_{2}^{\prime}\right)
\end{array}\right]
$$

No exemplo considerado, $\mathbf{q}_{1}^{\prime}$ contém os $J$ primeiros coeficientes de $\mathbf{q}_{1}$ e $\mathbf{q}_{2}^{\prime}$ contém os $J$ últimos coeficientes de $\mathbf{q}_{2}$. Em (12) se caracteriza o uplink e permite realizar uma equalização simplificada devido à presença da matriz diagonal (como em (7)). 


\section{EqualizaÇão E DeteCÇão Para CS-CDMA}

Na recepção, o sinal CS-CDMA, dependendo do intervalo de guarda empregado, é equalizado utilizando diferentes matrizes. $\mathrm{O}$ desenvolvimento feito nessa seção para o processo de equalização considera as duas opções: $\mathrm{CP}$ ou ZP. Sendo a matriz $\mathbf{G}_{k}$ a representação do canal para ambos os intervalos de guarda, (2) e (4) podem ser expressas de forma unificada por

$$
\mathbf{R}=\sum_{k=1}^{K} \mathbf{G}_{k} \mathbf{b}_{k} \mathbf{c}_{k}^{T}+\mathbf{N},
$$

onde $\mathbf{G}_{k}=\operatorname{Circ}\left(\overline{\mathbf{h}}_{k}\right)$ se é empregado CP ou $\mathbf{G}_{k}=\overline{\mathbf{H}}_{\mathbf{k}}^{(\mathbf{0})}$ no caso ZP. Ressalta-se que em ambos os casos os sistemas CS-CDMA permitem desacoplar idealmente os sinais dos diferentes usuários na recepção, eliminando portanto a MultipleAccess Interference (MAI), mesmo quando a transmissão é feita através de canais dispersivos no tempo e seletivos em frequência. O processo de desacoplamento pode ser feito antes da etapa de estimação de canal (se necessária), equalização e outros processos de detecção. Por exemplo, considerando códigos ortonormais e sendo $\mathbf{c}_{m}$ o código atribuído ao $\mathrm{m}$ ésimo usuário, resulta de (14) que o sinal correspondente a este usuário pode ser obtido por

$$
\mathbf{r}_{m}=\mathbf{R} \mathbf{c}_{m}=\mathbf{G}_{m} \mathbf{b}_{m}+\mathbf{n}_{m}
$$

onde $\mathbf{n}_{m}=\mathbf{N c}_{m}$ é um vetor de ruído aditivo Gaussiano branco com media zero e matriz covariância $\mathbb{E}\left[\mathbf{n}_{m} \mathbf{n}_{m}^{H}\right]=$ $\left\|\mathbf{c}_{m}\right\|^{2} \sigma^{2} \mathbf{I}=\sigma^{2} \mathbf{I}$. Em (15) se evidencia a ausência de componentes MAI devido à preservação da ortogonalidade dos sinais mesmo após propagação por canais que sofrem multipercursos.

Após a separação dos sinais dos diferentes usuários, equalizadores lineares ZF ou MMSE podem ser utilizados com as respectivas matrizes dadas por

$$
\begin{gathered}
\overline{\mathbf{A}}_{Z F}^{(m)}=\mathbf{G}_{m}^{\dagger}, \\
\overline{\mathbf{A}}_{M M S E}^{(m)}=\left[\mathbf{G}_{m}^{H} \mathbf{G}_{m}+\sigma^{2} \mathbf{I}\right]^{-1} \mathbf{G}_{m}^{H},
\end{gathered}
$$

onde $\mathbf{G}_{m}^{\dagger}=\mathbf{G}_{m}^{-1}$ no caso CP. No caso ZP, como a matriz $\overline{\mathbf{H}}_{\mathbf{m}}^{(\mathbf{0})}$ não é quadrada, $\mathbf{G}_{m}^{\dagger}$ corresponde à pseudo-inversa a esquerda desta matriz, $\mathbf{G}_{m}^{\dagger}=\left(\overline{\mathbf{H}}_{\mathbf{m}}^{(\mathbf{0}) \mathbf{H}} \overline{\mathbf{H}}_{\mathbf{m}}^{(\mathbf{0})}\right)^{-1} \overline{\mathbf{H}}_{\mathbf{m}}^{(\mathbf{0}) \mathbf{H}}$. Após a equalização de $\mathbf{r}_{m}$ em (15) a detecção dos componentes de $\mathbf{b}_{m}$ é feita por mínima distância aos pontos da constelação de sinais, dos componentes do vetor equalizado.

\section{Equalização e Detecção Para OFdMA}

Como foi suposto que as características estatísticas do canal são mantidas durante a transmissão de um bloco OFDMA, o processo de equalização após a DFT é simplificado. Cada subsímbolo requer de um equalizador de apenas um tap visando eliminar as distorções multiplicativas provocadas pelo canal. A complexidade da equalização é bem simples devido à matriz diagonal que após processamento caracteriza a ação do canal.

Os equalizadores de Zero Forcing (ZF) e Minimum Mean Squared Error (MMSE) supõem o conhecimento ideal do canal de propagação na recepção. Considerando a adição de $\mathrm{CP}$, que os sub-símbolos tenham energia unitária e que o vetor de ruído $\mathbf{n}$ possui uma matriz de covariância $\mathbf{K}_{n}=\sigma^{2} \mathbf{I}_{N}$, as matrizes correspondentes aos equalizadores são dadas por [11]

$$
\mathbf{A}_{Z F}^{c p}=\operatorname{Diag}(\mathbf{q})^{-1}
$$

$$
\mathbf{A}_{M M S E}^{c p}=\operatorname{Diag}(\mathbf{q})^{H}\left[\operatorname{Diag}(\mathbf{q}) \operatorname{Diag}(\mathbf{q})^{H}+\sigma^{2} \mathbf{I}_{N}\right]^{-1},
$$

com $\mathbf{A}_{Z F}^{c p}$ e $\mathbf{A}_{M M S E}^{c p}$ representando as matrizes de ZF e MMSE, respectivamente, com dimensão $N \times N$, que multiplicam as observações (12) antes da detecção de sinais, implementada por mínima distância aos pontos da constelação de sinais, dos componentes do vetor equalizado.

\section{RESUlTAdos NumÉRICOS}

Para uma justa comparação considera-se que os sistemas de múltiplo acesso tenham a mesma resposta impulsional do canal discreto $\left(L=L_{0}\right)$ e a mesma largura de banda de transmissão. Além disso, considera-se que os sistemas transmitem o mesmo número de símbolos de informação $\left(N=K * B_{0}\right)$ e contam com igual número de usuários ativos. Para a análise de desempenho entre as duas técnicas foram obtidos curvas de BER (Bit-Error Rate) em função da relação $E_{B} / N_{0} \mathrm{em} \mathrm{dB}$, onde $E_{B}$ denota a Energia por bit de informação transmitido.

Para gerar as curvas foi utilizada a modulação QPSK e os códigos ortogonais binários de Hadamard de comprimento $T_{0}$. Os canais associados aos $K$ usuários do sistema são aleatórios, estatisticamente independentes, identicamente distribuídos e modelados por filtros FIR com $P$ coeficientes. Os coeficientes do $k$-ésimo canal de propagação, $\mathbf{h}_{k}$, são dados por $h_{k_{i}}=$ $p_{i} \alpha_{k_{i}}$, onde $\left\{\alpha_{k_{i}}\right\}_{i=0}^{P-1}$ são variáveis aleatórias Gaussianas complexas circularmente simétricas, estatisticamente independentes com média nula e $\mathbb{E}\left[\left|\alpha_{k_{i}}\right|^{2}\right]=1$. Os valores de $\alpha_{k_{i}}$ são gerados aleatoriamente e mantidos fixos em cada simulação. Os pesos $p_{i}$ satisfazem $\sum_{i=0}^{P-1}\left|p_{i}\right|^{2}=1$, tal que $\mathbb{E}\left[\left\|\mathbf{h}_{k}\right\|^{2}\right]=$ 1. O intervalo de guarda foi considerado com um comprimento suficiente para eliminar a IEB $(L \geq P-1)$.

Inicialmente é interessante comparar o comportamento do sistema CS-CDMA com a faixa de guarda do tipo CP e com a do tipo ZP, sugerida e analisada neste artigo. A Figura 2 mostra as curvas de desempenho do sistema CS-CDMA em configuração de uplink para os equalizadores ZF e MMSE, implementados com os dois diferentes tipos de intervalo de guarda. Para a simulação se utilizou $T_{0}=8, B_{0}=8$, 8 usuários ativos e um canal com $P=4$ e pesos $p_{0}=$ $0.8867, p_{1}=0.4116, p_{2}=0.1910, p_{3}=0.0887$.

Como ilustra a Figura 2, o emprego do ZP como intervalo de guarda obtém melhores resultados de desempenho. Um fator que incide nos resultados atingidos pelo sistema com ZP, é que a matriz que caracteriza ao canal $\left(\overline{\mathbf{H}}_{\mathbf{k}}^{(\mathbf{0})}\right)$, devido a adição de zeros no final do vetor de dados, fica de dimensão $M_{0} \times B_{0}$ onde $M_{0}>B_{0}$. A interpretação desse fato, corresponde a que o problema tem então mais equações que incógnitas, permitindo obter melhores soluções. Além disso, a utilização do intervalo de guarda ZP permite que a energia despendida pelo sistema para transmitir os símbolos que formam o intervalo de guarda seja nula, uma vez que nenhum sinal é transmitido durante este intervalo. Desta forma a energia por símbolo transmitido se iguala à energia por símbolo de informação 


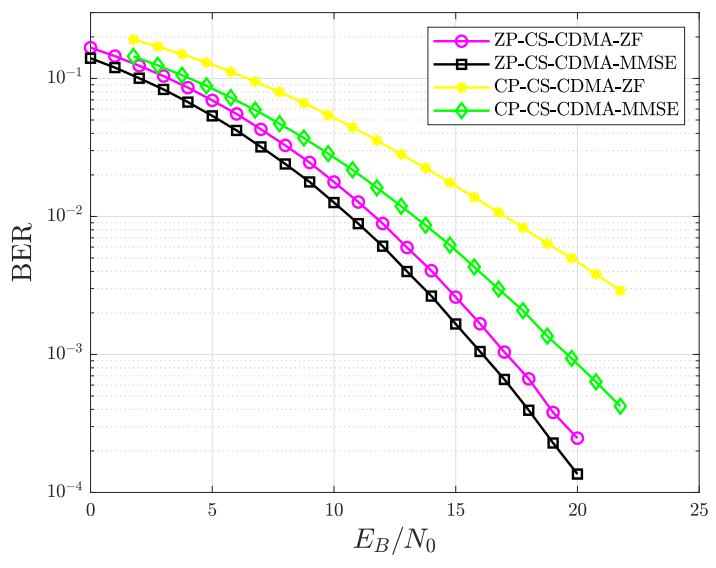

Fig. 2. BER vs $E_{B} / N_{0}$ para sistemas CS-CDMA com intervalos de guarda ZP e CP e parâmetros $L_{0}=4, B_{0}=8, K=T_{0}=8$ e $P=4$. Configuração uplink.

transmitido, ao contrário do $\mathrm{CP}$ que emprega energia para transmitir os símbolos redundantes presentes no intervalo de guarda.

A Figura 3 apresenta uma comparação de desempenho entre os sistemas CS-CDMA e o tradicional OFDMA, ambos em configuração de uplink. Os parâmetros considerados para obter os gráficos foram para o sistema CS-CDMA, os mesmos utilizados na geração da Figura 2 e para o sistema OFDMA se fixaram $\mathrm{N}=64$ subportadoras com faixa de guarda $\mathrm{CP}$.

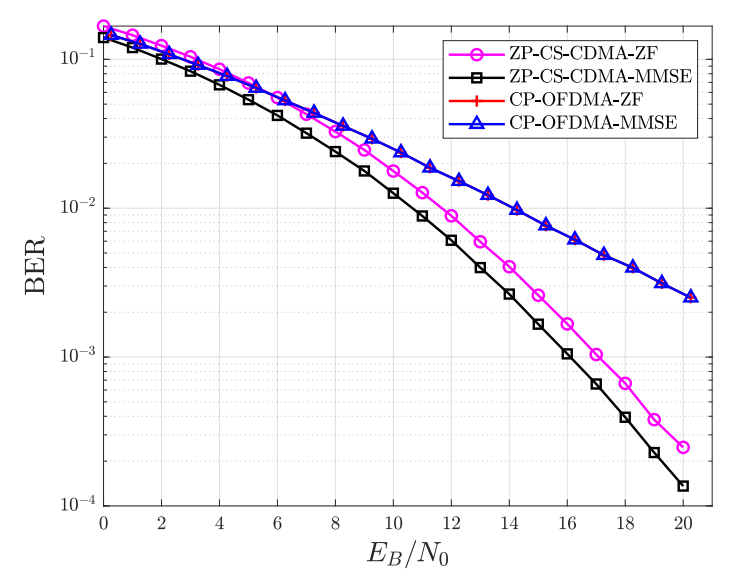

Fig. 3. BER vs $E_{B} / N_{0}$ para sistemas OFDMA e CS-CDMA com intervalos de guarda ZP e CP e parâmetros $L_{0}=4, B_{0}=8, K=T_{0}=8$ e $P=4$. Configuração uplink.

A Figura 3 ilustra as curvas de desempenho dos sistemas, empregando os equalizadores ZF e MMSE. Os resultados evidenciam a grande superioridade do desempenho do sistema CS-CDMA quando comparado com o obtido com o sistema OFDMA. Apesar de não apresentado diretamente na Figura 3, por falta de espaço, os resultados indicam um impressionante ganho de $14 \mathrm{~dB}$ para uma BER de $10^{-4}$, com este ganho aumentando rapidamente para valores menores de BER. Para o caso implementado neste artigo, o número de usuários é o máximo suportado pelo sistema CS-CDMA, quando utiliza códigos ortogonais. Para aumentar este número de usuários sem a ocorrência de MAI, o comprimento da sequência de espalhamento $\left(T_{0}\right)$ deve ser aumentado, assim como o número $N$ de subportadoras no caso OFDMA.

\section{CONClusões}

Este artigo enfocou o esquema de múltiplo acesso CSCDMA, incluindo neste estudo uma comparação entre a utilização de faixas de guarda do tipo $\mathrm{CP}$ e o tipo ZP, proposta aqui, onde alguns fatores que levaram a adição do ZP a apresentar melhores resultados foram observados. Foi também enfatizado a principal vantagem dos sistemas CSCDMA sobre os sistemas CDMA tradicionais, aquela que permite que os usuários sejam desacoplados idealmente na recepção mesmo quando a transmissão é feita através de canais dispersivos no tempo e seletivos em frequência. Como medida da eficácia do esquema CS-CDMA como técnica de acesso múltiplo foram incluídas comparações de desempenho com o tradicional esquema OFDMA que indicaram uma larga superioridade do sistema CS-CDMA. Outras comparações entre estes sistemas envolvendo densidades espectrais de potencia, eficiência espectral e comportamento em canais não lineares podem ser encontradas em [12].

\section{REFERÊNCIAS}

[1] K. Fazel e S. Kaiser, "Multi-Carrier and Spread Spectrum Systems: From OFDM and MC-CDMA to LTE and WiMAX," Wiley, 2008.

[2] A. Chouly, A. Brajal e S. Jourdan, "Orthogonal multicarrier techniques applied to direct sequence spread spectrum CDMA systems," Proceedings of GLOBECOM '93. IEEE Global Telecommunications Conference, v.3, pp. 1723-1728, 1993.

[3] V. DaSilva e E. S. Sousa, "Performance of orthogonal CDMA codes for quasi-synchronous communication systems," Proceedings of 2nd IEEE International Conference on Universal Personal Communications, v.2, pp. 995-999, 1993.

[4] L. L. Yang e L. Hanzo, "Multicarrier DS-CDMA: a multiple access scheme for ubiquitous broadband wireless communications," IEEE Communications Magazine, v. 41, n. 10, pp. 116-124, Outubro 2003.

[5] A. Madhukumar, F. Chin, Y. Liang e K. Yang, "Single Carrier Cyclic Prefix-Assisted CDMA System with Frequency Domain Equalization for High Data Rate Transmission," EURASIP J. Wireless Communication Network, pp. 149-160, 2004

[6] K. L. Baum, T. A. Thomas, F. W. Vook e V. Nangia, "Cyclic-prefix CDMA: an improved transmission method for broadband DS-CDMA cellular systems," IEEE Wireless Communications and Networking Conference Record., v.1, pp. 183-188, 2002.

[7] L. Hanzo, L. L. Yang, E. L. Kuan e K. Yen, "Single- and Multi-Carrier DS-CDMA: Multi-User Detection, Space-Time Spreading, Synchronization, Networking and Standards," Wiley, 2003.

[8] L. Arevalo, C. A. Medina e R. Sampaio-Neto, "Playing with Blocks: A New Combination of Block Transmission and the CDMA Multiple Access Technique," IEEE 81st Vehicular Technology Conference (VTC Spring), pp. 1-4, Maio 2015.

[9] D. Pereira, L. Arevalo, R. P. David e R. Sampaio-Neto, "Chip-Spread CDMA Transmission: A Comparative Analysis," XXXIV Simpósio Brasileiro de Telecomunicações e Processamento de Sinais - SBrT 2016, pp. 428-432, 2016.

[10] J. Li, X. Wu e R. Laroia, "OFDMA Mobile Broadband Communications: A Systems Approach," Cambridge University Press, 2013.

[11] R. P. David, "Técnica de Estimação de Canal utilizando Símbolos Piloto em Sistemas OFDM," Dissertação de Mestrado, Pontifícia Universidade Católica do Rio de Janeiro, 2007.

[12] E. Rodríguez-Hernández, "Orthogonal Multiple Access Schemes in Linear and Non-linear Channels," Dissertação de Mestrado, Pontifícia Universidade Católica do Rio de Janeiro, 2021. 